Communications in Physics, Vol.22, No.1 (2012), pp. 45-51

\title{
TEMPERATURE EFFECTS ON THE PLASMON MODES OF DOUBLE-LAYER GRAPHENE
}

\author{
DINH VAN TUAN, NGUYEN QUOC KHANH \\ Department of Theoretical Physics, Ho Chi Minh City National University
}

\begin{abstract}
We calculate the dynamical dielectric function of doped double-layer graphene (DLG), made of two parallel graphene monolayers with carrier densities $n_{1}, n_{2}$, respectively, and a separation interlayer of $d$ at finite temperature. The results are used to find the dispersion of plasmon modes. We study the temperature effects on the DLG plasmon modes in the case of symmetric system $\left(n_{1}=n_{2}\right)$, asymmetric system $\left(n_{1} \neq n_{2}\right)$ and no free carriers in the second layer $\left(n_{2}=0\right)$. We show that the effect of temperature on the plasmon dispersion is significant and can not be ignored in investigating graphene properties.
\end{abstract}

\section{INTRODUCTION}

Graphene is a two-dimensional electron system that has attracted a great deal of attention because of its unique electron properties [1] and its potential as a new material for electronic technology $[2,3]$. The main difference of $2 \mathrm{D}$ graphene compared with 2D semiconductor system is the electronic energy dispersion. In 2D semiconductor systems, the electron energy depends quadratically on the momentum, but in graphene, the dispersions of electron are linear near K, K' points of the Brillouin zone [4]. Because of the difference of electronic band structure, there are many graphene properties which are significantly different from the behavior of electrons in the ordinary 2D systems [5].

In this paper, we consider the temperature effects on the plasmon mode of a DLG system formed by two parallel single-layer graphene (SLG) separated by a distance $d$. The DLG is fundamentally different from the well-studied bilayer graphene [6] because there is no inter-layer tunneling, only inter-layer Coulomb interaction. Spatially separated twocomponent DLG can be fabricated by folding an SLG over a high-insulating substrate [7].

Recently, Hwang and Das Sarma [8] have investigated the plasmon dispersion in doped DLG at zero temperature and found the surprising results. They have shown that the plasmon modes of an interacting DLG system are completely different from the doublelayer semiconductor quantum well plasmons. In the long-wavelength limit the density dependence of plasmon frequency is given by $\left(\omega_{0}^{+}\right)^{2} \propto \sqrt{n_{1}}+\sqrt{n_{2}}$ for optical plasmon and $\left(\omega_{0}^{-}\right)^{2} \propto \sqrt{n_{1}} \sqrt{n_{2}} /\left(\sqrt{n_{1}}+\sqrt{n_{2}}\right)$ for acoustic plasmon compared to $\left(\omega_{0}^{+}\right)^{2} \propto N$ and $\left(\omega_{0}^{+}\right)^{2} \propto n_{1} n_{2} / N$ in ordinary $2 \mathrm{D}$ systems, where $N=n_{1}+n_{2}$ 


\section{THEORY}

In graphene, the energy dispersion near the $\mathrm{K}, \mathrm{K}^{\prime}$ points of the Brillouin zone is given by $[9]$ :

$$
\epsilon_{\mathbf{k}, s}=s \nu_{F}|\mathbf{k}|,
$$

where $s= \pm 1$ indicate the conduction $(+1)$ and valance $(-1)$ bands, respectively, and $\nu_{F}$ is the Fermi velocity of graphene and $\hbar=1$ throughout this paper. The Fermi momentum $\left(k_{F}\right)$ and the Fermi energy $\left(E_{F}\right)$ of $2 \mathrm{D}$ graphene are given by $k_{F}=\sqrt{4 \pi n / g}$ and $E_{F}=$ $\nu_{F} k_{F}$ where $n$ is the $2 \mathrm{D}$ carrier density and $g=g_{s} g_{\nu}=4$ accounts for the spin $\left(g_{s}=2\right)$ and valley $\left(g_{\nu}=2\right)$ degeneracy.

In RPA, the dynamical dielectric function of SLG becomes

$$
\varepsilon(q, \omega, T)=1-v_{c}(q) \Pi(q, \omega, T),
$$

where $v_{c}(q)=2 \pi e^{2} / \kappa q$ is the 2D Fourier transform of the Coulomb potential and $\Pi(q, \omega, T)$, the $2 \mathrm{D}$ polarizability at finite temperature, is given by the bare bubble diagram [10]

$$
\Pi(q, \omega, T)=g \lim _{\eta \rightarrow 0^{+}} \sum_{s, s^{\prime}= \pm 1} \int \frac{d^{2} \mathbf{k}}{(2 \pi)^{2}} \frac{1+s s^{\prime} \cos \left(\theta_{\mathbf{k}, \mathbf{k}+\mathbf{q}}\right)}{2} \frac{n_{F}\left(\epsilon_{\mathbf{k}, s}\right)-n_{F}\left(\epsilon_{\mathbf{k}+\mathbf{q}, s^{\prime}}\right)}{\omega+\epsilon_{\mathbf{k}, s}-\epsilon_{\mathbf{k}+\mathbf{q}, s^{\prime}}+i \eta},
$$

Here $n_{F}(\epsilon)=\left\{\exp \left[\beta\left(\epsilon-\mu_{0}\right)\right]+1\right\}^{-1}$ is the Fermi-Dirac distribution function, $\mu_{0}=\mu_{0}(T)$ being the noninteracting chemical potential determined by the conservation of the total electron density as

$$
\frac{1}{2}\left(\frac{T_{F}}{T}\right)^{2}=F_{1}\left(\beta \mu_{0}\right)+F_{1}\left(-\beta \mu_{0}\right),
$$

where $\beta=1 / k_{B} T$ and $F_{n}(x)$ is given by

$$
F_{n}(x)=\int_{0}^{\infty} \frac{t^{n} d t}{1+\exp (t-x)}
$$
by [11]

The limiting forms of the chemical potential in low and high temperature are given

$$
\begin{array}{rlrl}
\mu_{0}(T) & \approx E_{F}\left[1-\frac{\pi^{2}}{6}\left(\frac{T}{T_{F}}\right)^{2}\right], & \frac{T}{T_{F}} & \ll 1 \\
\mu_{0}(T) & \approx \frac{E_{F}}{4 \ln 2} \frac{T_{F}}{T}, & \frac{T}{T_{F}} \gg 1 .
\end{array}
$$

Recently, MacDonald and coworkers [12] have presented the following semi-analytical expressions for the imaginary $[\operatorname{Im} \Pi(q, \omega, T)]$ and the real $[\operatorname{Re} \Pi(q, \omega, T)]$ parts of the dynamical polarizability:

$$
\begin{aligned}
\operatorname{Im} \Pi(q, \omega, T)= & \frac{g}{4 \pi} \sum_{\alpha \pm}\left\{\Theta\left(\nu_{F} q-\omega\right) q^{2} f\left(\nu_{F} q, \omega\right)\left[G_{+}^{(\alpha)}(q, \omega, T)-G_{-}^{(\alpha)}(q, \omega, T)\right]+\right. \\
& \left.+\Theta\left(\omega-\nu_{F} q\right) q^{2} f\left(\omega, \nu_{F} q\right)\left[-\frac{\pi}{2} \delta_{\alpha,-}+H_{+}^{(\alpha)}(q, \omega, T)\right]\right\}
\end{aligned}
$$



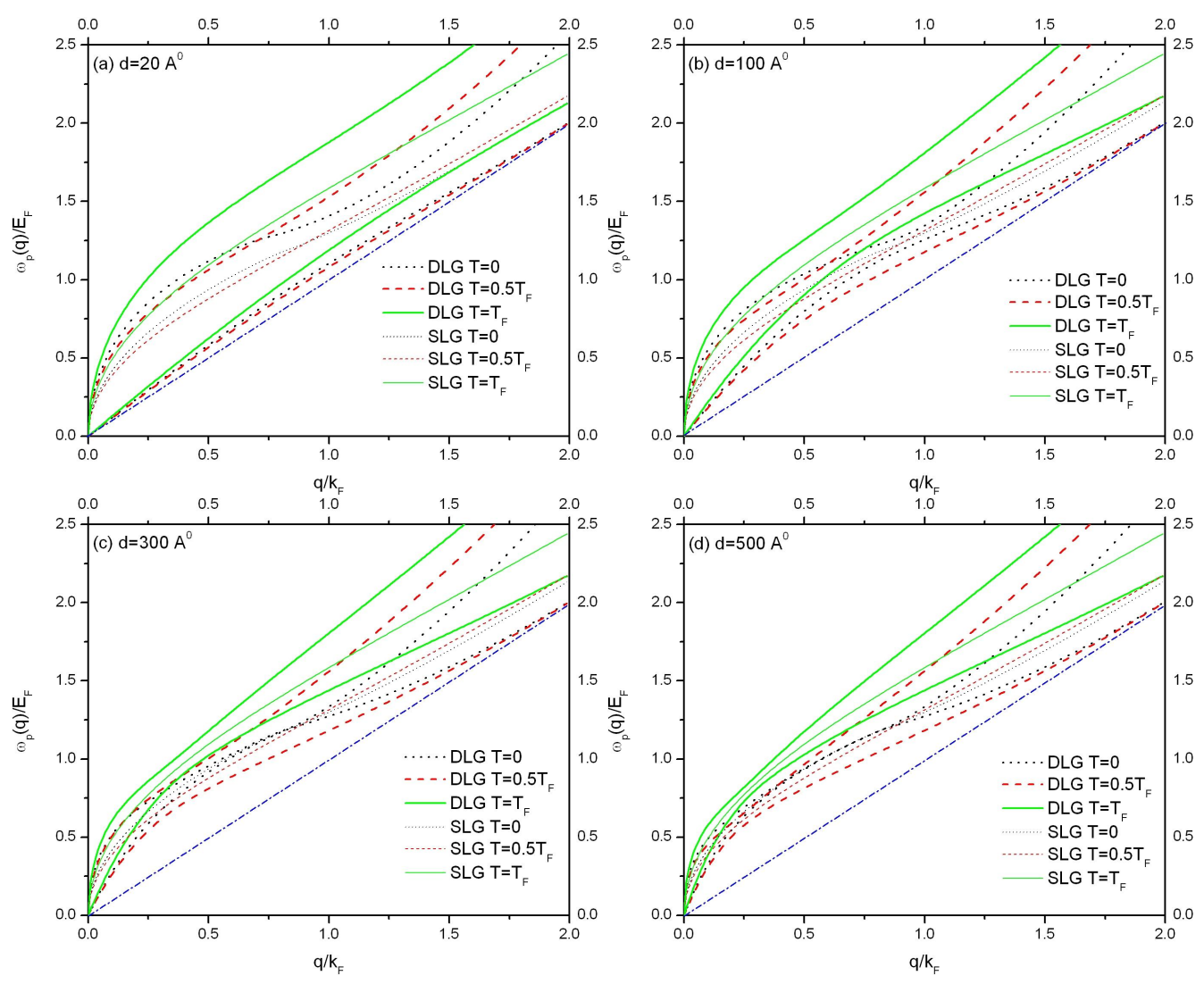

Fig. 1. Plasmon dispersions of DLG for several layer separations at $T=0$ (bold dotted lines), $T=0.5 T_{F}$ (bold dashed lines) and $T=T_{F}$ (bold solid lines). The thin lines indicate the plasmon dispersion of SLG with the same density and temperature. Here we use the parameters: $n_{1}=n_{2}=10^{12} \mathrm{~cm}^{-2}$ and (a) $d=$ $20 \AA\left(k_{F} d=0.35\right),(\mathrm{b}) d=100 \AA\left(k_{F} d=1.8\right),(\mathrm{c}) d=300 \AA\left(k_{F} d=5.3\right)$ and $(\mathrm{d}) d=$ $500 \AA\left(k_{F} d=8.9\right)$

$$
\begin{aligned}
\operatorname{Re} \Pi(q, \omega, T)= & \frac{g}{4 \pi} \sum_{\alpha \pm}\left\{\frac{-2 k_{B} T \ln \left[1+e^{\alpha \mu_{0} /\left(k_{B} T\right)}\right]}{\nu_{F}^{2}}+\right. \\
& +\Theta\left(\omega-\nu_{F} q\right) q^{2} f\left(\omega, \nu_{F} q\right)\left[G_{-}^{(\alpha)}(q, \omega, T)-G_{+}^{(\alpha)}(q, \omega, T)\right]+ \\
& \left.+\Theta\left(\nu_{F} q-\omega\right) q^{2} f\left(\nu_{F} q, \omega\right)\left[-\frac{\pi}{2} \delta_{\alpha,-}+H_{-}^{(\alpha)}(q, \omega, T)\right]\right\}
\end{aligned}
$$

where

$$
\begin{gathered}
f(x, y)=\frac{1}{2 \sqrt{x^{2}-y^{2}}}, \\
G_{ \pm}^{(\alpha)}(q, \omega, T)=\int_{1}^{\infty} d u \frac{\sqrt{u^{2}-1}}{\exp \left(\frac{\left|\nu_{F} q u \pm \omega\right|-2 \alpha \mu_{0}}{2 k_{B} T}\right)+1},
\end{gathered}
$$




$$
H_{ \pm}^{(\alpha)}(q, \omega, T)=\int_{-1}^{1} d u \frac{\sqrt{1-u^{2}}}{\exp \left(\frac{\left|\nu_{F} q u \pm \omega\right|-2 \alpha \mu_{0}}{2 k_{B} T}\right)+1} .
$$

The DLG dielectric function is obtained from the determinant of the generalized dielectric tensor and has the following form

$$
\varepsilon_{D L G}(q, \omega, T)=\varepsilon_{1}(q, \omega, T) \varepsilon_{2}(q, \omega, T)-v_{12}(q) v_{21}(q) \Pi_{1}(q, \omega, T) \Pi_{2}(q, \omega, T) .
$$

Here $\varepsilon_{1}(q, \omega, T)$ and $\varepsilon_{2}(q, \omega, T)$ are the dynamical dielectric functions of individual layers given by the Eq.(2), $v_{12}(q)=v_{21}(q)=2 \pi e^{2} \exp (-q d) /(\kappa q)$, with $\kappa$ is the background lattice dielectric constant, are the interlayer Coulomb interaction matrix elements.

The spectrum of the collective excitations is obtained from the zeros of the real part and the imaginary part of the double-layer dielectric function describes the damping of collective modes.

\section{NUMERICAL RESULTS}

\section{III.1. Symmetric System}

As shown in Fig. 1, the frequency of the acoustic mode $\omega_{-}$decreases compared with the SLG plasmon mode at the same temperature, while the optical mode $\omega_{+}$shifts to higher energy. For low temperatures, the acoustic mode $\omega_{-}$approaches the $\omega=\nu_{F} q$ line (dotdashed line) in the high-energy region. More interestingly, when $T<T_{C}\left(T_{C} \approx 0.6 T_{F}\right)$, the acoustic mode $\omega_{-}$is below that at zero temperature, while for $T>T_{C}$, both acoustic and optical modes are above those at zero temperature. Furthermore, as $T$ increases $\left(T>T_{C}\right)$ both acoustic and optical modes shift to higher energy. As $d$ increases both the acoustic mode $\omega_{-}$and the optical mode $\omega_{+}$in the low-frequency region approach the SLG plasmon at the same temperature. For large momentum $\left(q>k_{F}\right)$, we find that the mode dispersion is unchanged when $d>100 \AA$.

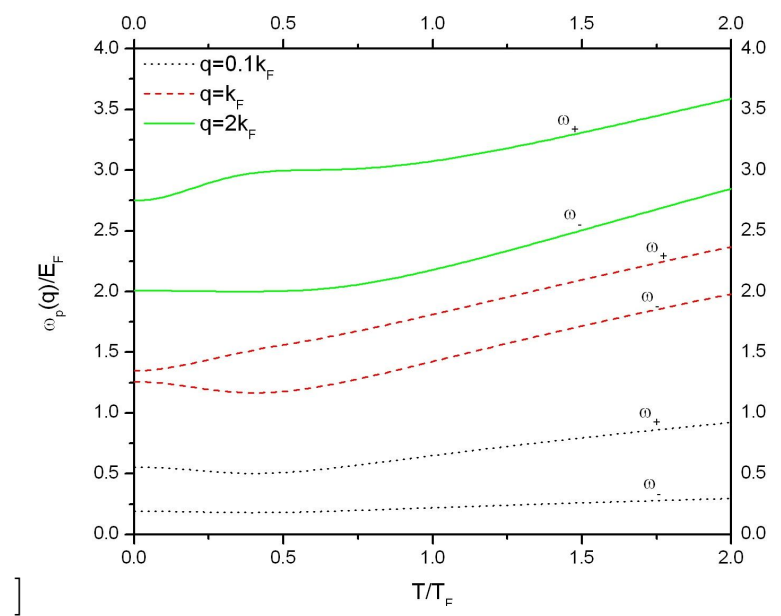

Fig. 2. The plasmon modes of DLG at several momentum values and fixed layer separation $d=100 \AA$ 
In Fig. 2, we show the temperature effect on the plasmon mode dispersion at several momentum values and fixed layer separation $d=100 \AA$. When $T<T_{0}\left(T>T_{0}\right)\left(T_{0} \approx\right.$ $\left.0.4 T_{F}\right)$ the acoustic mode $\omega_{-}$decreases (increases) when the temperature $T$ increases.

The Fig. 2 shows that in the case of low momentum, the optical mode $\omega_{+}$decreases and then increases while it only increases in the cases of large momentum. The Fig. 2 also shows that the high temperature plasmon modes are dramatically different from the zero temperature ones especially in the case of large momentum.
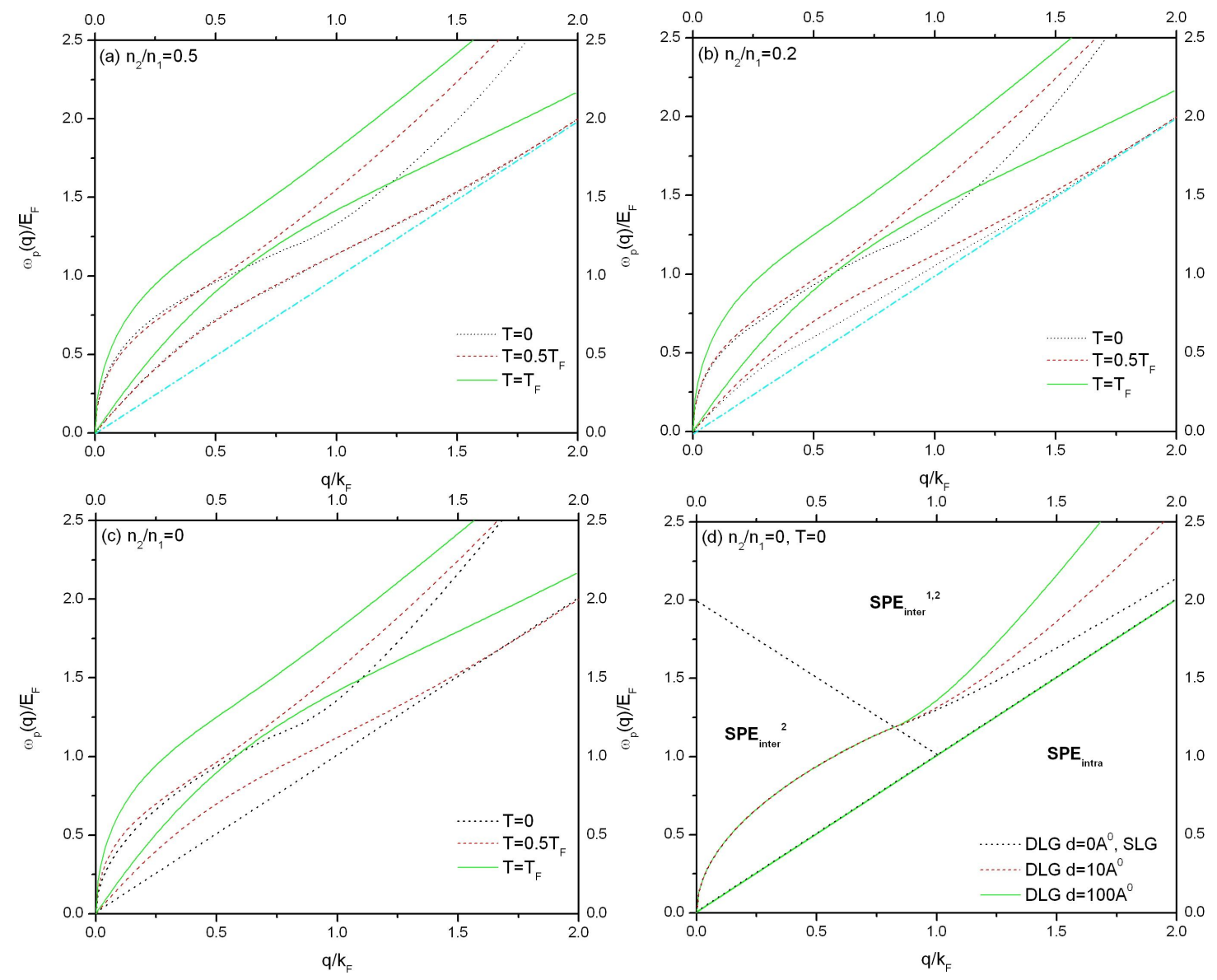

Fig. 3. Plasmon dispersions of DLG for different temperatures and layer densities. Here we use $n_{1}=10^{12} \mathrm{~cm}^{-2}$ and $d=100 \AA$

\section{III.2. Asymmetric System}

In Fig. 3 we observe that the plasmon dispersion is almost unchanged at high temperature $\left(T \approx T_{F}\right)$. As $n_{2} / n_{1}$ decreases, the zero temperature acoustic mode $\omega_{-}$ approaches the boundary of the intraband single-particle excitation $\left(S P E_{\text {intra }}\right)$ but it shifts, however, to higher energy when the temperature increases. In Fig. 3(d) we show the DLG plasmon modes at zero temperature for several separations in the case of $n_{2} / n_{1}=0$, 
i.e., the second layer is undoped and the first layer has a finite density $n_{1}=10^{12} \mathrm{~cm}^{-2}$. It is seen from the figure that the acoustic mode $\omega_{-}$is degenerate with the boundary of $S P E_{\text {intra }}$ (i.e. $\omega=\nu_{F} q$ ), while the optical mode $\omega_{+}$is degenerate with the SLG optical mode below the $S P E_{\text {intra }}^{1,2}$. As $d \rightarrow 0$ the dispersion of $\omega_{+}$becomes exactly that of SLG plasmon with the same density.
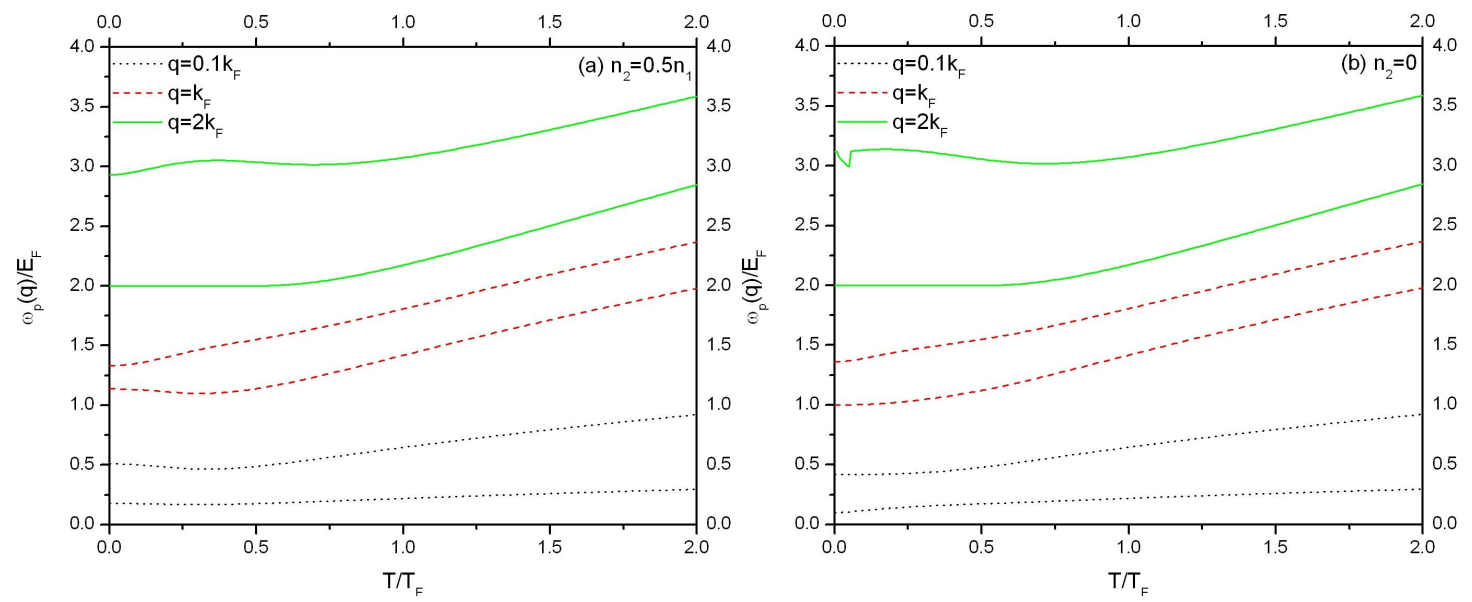

Fig. 4. The DLG plasmon modes for unequal densities at several momentum values and fixed layer separation $d=100 \AA$

As shown in Fig. 4, the acoustic mode $\omega_{-}$ships to low energy at low temperature when $n_{2} / n_{1}$ decreases. The DLG plasmon modes are unchanged at high temperature. In the low temperature and large momentum region, the acoustic mode $\omega_{-}$approaches the $\omega=\nu_{F} q$ line. The Fig. 4(b) also show that the optical plasmon mode $\omega_{-}$for $n_{2}=0$ and $q=2 k_{F}$ suddenly drops and then increases at low temperature $\left(T \approx 0.05 T_{F}\right)$

\section{CONCLUSIONS}

In this paper, we have investigated the temperature effect on the plasmon dispersion mode of doped DLG, made of two parallel graphene monolayers with carrier densities $n_{1}, n_{2}$, and a separation interlayer of $d$. Our results show that the effect of temperature on the plasmon dispersion is significant and can not be ignored in investigating many graphene properties.

\section{ACKNOWLEDGMENT}

We thank Do Hoang Son and Nguyen Thanh Son for useful discussions. This work is supported by the Vietnam's National Foundation for Science and Technology Development (NAFOSTED). 


\section{REFERENCES}

[1] H. Castro Neto, F. Guinea, N. M. R. Peres, K. S. Novoselov, A. K. Geim, Rev. Mod. Phys. , 81 (2009) 109.

[2] A. K. Geim, K. S. Novoselov, Nature Mater 6 (2007) 183.

[3] A. K. Geim, A. H. Macdonald, Phys. Today 60 (2007) 35.

[4] P. R. Wallace, Phys. Rev. 71 (1947) 622.

[5] E. H. Hwang, S. Das Sarma, Phys. Rev. B75 (2007)205418.

[6] E. McCann, V. I. Fal'ko, Phys. Rev. Lett. 96 (2006) 086805;

[7] H. Schmidt, T. Ludtke, P. Barthold, E. McCann, V. I. Fal'ko, R. J. Haug, Appl. Phys. Lett. 93 (2008) 172108 .

[8] E. H. Hwang, S. Das Sarma, Phys. Rev. B80 (2009) 205405.

[9] J. C. Slonczewski, P. R. Weiss, Phys. Rev. 109 (1958) 272;

[10] B. Wunsch, T. Stauber, F. Sols, F. Guinea, New J. Phys. 8 (2006) 318.

[11] E. H. Hwang, S. Das Sarma, Phys. Rev. B79 (2009) 165404.

[12] M. R. Ramezanali, M. M. Vazifeh, R. Asgari, M. Polini, A. H. MacDonald, J. Phys. A: Math. Theor. 42 (2009) 214015.

Received 15 December 2010. 